\title{
Epworth Sleepiness Scale- a novel tool to assess somnolence syndrome in patients receiving radiotherapy to the brain
}

\author{
Ritika Rajkumar Harjani ${ }^{1}$, Manur Gururajachar Janaki' ${ }^{1}$ Uma Maheswari Krishnaswamy², \\ Ram Charith Alva ${ }^{1}$, Arul Ponni ${ }^{1}$, Kirthi Koushik ${ }^{1}$, Mohan Kumar Somashekar1, \\ Ram Abhinav Kannan ${ }^{1}$, Arvind Sathya Murthy ${ }^{1}$ \\ ${ }^{I}$ Department of Radiotherapy, MS Ramaiah Medical College, Bangalore, India \\ ${ }^{2}$ Department of Chest and Sleep Medicine, M S Ramaiah Medical College, Bangalore, India
}

Received September 29, 2014; Revised November 22, 2014; Accepted November 26, 2014; Published Online November 29, 2014

\section{Original Article}

\begin{abstract}
Purpose: Radiation to brain causes early, early-delayed, and delayed side effects. There is paucity of literature regarding early-delayed effects like somnolence syndrome. Existing studies use general symptom assessment and visual analog scales. Epworth Sleepiness Scale (ESS) is a time tested tool to assess daytime sleepiness in various conditions. In this study, the ESS has been used to determine the occurrence of somnolence in patients receiving cranial radiotherapy for primary and metastatic brain tumors. Thus the ESS has been used in a novel setting in our study. The ESS is a simple to administer questionnaire and may be useful in grading the severity of somnolence. To our knowledge, this is the second study to determine post radiation somnolence using ESS. Methods: This prospective study was conducted in 23 patients with primary and metastatic brain tumor. Patient demographics and tumor type and grade was noted. Those with Karnofsky Performance Scale (KPS) less than 70 and with pre-existing sleep disorders were excluded. Radiotherapy regimen included palliative whole brain radiation for brain metastases and conformal adjuvant radiotherapy for primary brain tumors as per standard guidelines. All subjects included were administered ESS at baseline and weekly thereafter during and for 6 weeks after radiation. Results: All 23 patients (median age 50 years) completed the planned questionnaires until 6 weeks post radiation. Twenty (87\%) patients had primary brain tumors whereas three (13\%) patients had metastatic lesions in brain. Of the 23 patients, 14 patients (60.86\%) had abnormal or increased daytime sleepiness; of which 3 had ESS scores greater than 16. Conclusion: Somnolence was noted in $60.86 \%$ of the patients, which is in accordance with existing literature. Epworth sleepiness scale is an effective tool to detect and quantify somnolence, However, it does not consider other symptoms of somnolence syndrome and hence should be combined with visual analog scale to get complete information.
\end{abstract}

Keywords: Brain Malignancy; Epworth Sleepiness Scale; Radiation Therapy; Sleepiness; Somnolence Syndrome

\section{Introduction}

Malignant tumors of the brain are either primary or metastatic, the latter being more common. Metastases commonly arise from lung, breast, colo-rectum, etc. ${ }^{1}$ The primary tumors are most often neuro-epithelial in origin; namely astrocytomas, oligodendrogliomas, ependymomas, amongst others. As for any malignancy, the three broad treatment options are surgery, radiation therapy and chemotherapy. Radiation therapy forms the mainstay of treatment, either alone or in an adjuvant setting. In view of the blood brain barrier, chemotherapy has limited role, commonly as a radio-sensitizer in high grade gliomas. Radiotherapy plays the prime role in management of brain metastases. The most time tested modality is whole brain radiotherapy, commonly to a dose of 20-30 Gy in 5 to 10 fractions. Radiotherapy also plays a key role in primary brain tumors. The treatment in- volves maximal safe resection followed by adjuvant radiation to a dose of 54 to $60 \mathrm{~Gy}$ with or without chemotherapy depending upon histology and grade of the tumor.

Radiotherapy causes a number of adverse effects- and have been divided into acute, sub-acute and delayed. Acute effects occur during radiation and up to 6 weeks of completing radiation. They can present with transient worsening of pre-treatment deficits during radiotherapy or can manifest as general symptoms like fatigue, headache, and drowsiness. Late effects include neuro-cognitive dysfunction and brain necrosis.

Sub-acute or early delayed effects include head ache, fatigability and somnolence syndrome. Although early and de-

Corresponding author: Ritika Rajkumar Harjani; Department of Radiotherapy, M S Ramaiah Medical College, Bangalore, India. 
layed effects are well documented, Somnolence syndrome is less studied. Commonly, general symptom assessment and ranking on visual analog scale are undertaken to study this syndrome. ${ }^{2,3}$

Epworth Sleepiness Scale (ESS) is an instrument to measure day time sleepiness and is a useful tool to diagnose sleep disturbances. ${ }^{4}$ On the basis of scores obtained, propensity to sleep can be categorized and this can potentially help to quantify somnolence. This study is undertaken to study the incidence of somnolence in the patients in our department receiving radiotherapy to brain using Epworth Sleepiness Scale.

\section{Methods and Materials}

\section{Patient population}

This is a prospective study based on the patients with histologically proven primary brain tumors or brain metastases treated in our department with radiation therapy from January 2013 to December 2013. Patient demographics and tumor type- primary or metastatic along with grade was noted. Patients with Karnofsky performance status less than 70 and those with pre-existing sleep disorders were excluded from the study.

\section{Treatment}

Patients with primary brain tumors were subjected to initial surgery ranging from stereotactic biopsy to total decompression followed by radiation therapy to a dose of 5400 to 5940 cGy in conventional fractionation depending upon the histology and grade of the lesion. The post-op tumor bed along with residual disease with adequate margins were the volumes of interest. Metastatic brain tumors were treated with whole brain radiotherapy to a dose of $3000 \mathrm{cGy}$ in 10 fractions as per departmental protocol.

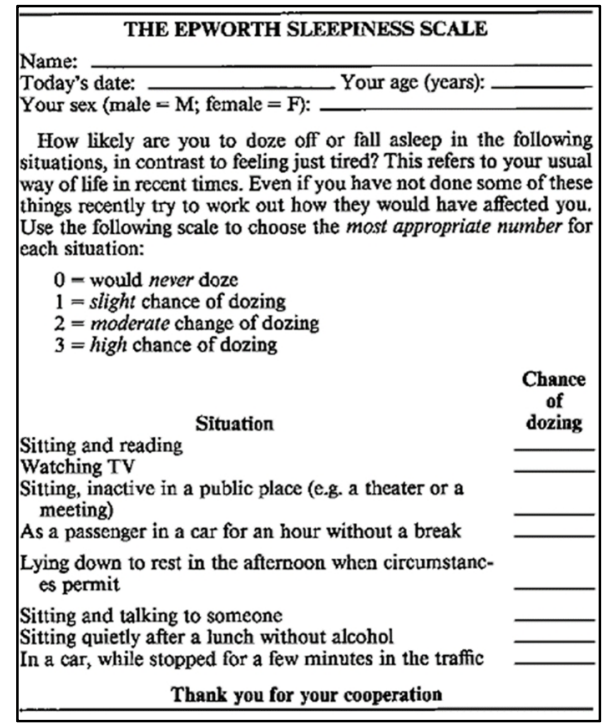

FIG. 1: Epworth Sleepiness Scale. ${ }^{4}$

\section{Assessment for somnolence}

Patients were subjected to Epworth Sleepiness Scale ${ }^{4}$ (Figure 1) at baseline, thereafter every week during radiation; also continuing weekly after cessation of radiation until 6 weeks post treatment.

\section{Results}

\section{Demography, Tumor characteristics and Performance status}

Twenty three patients were assessed and all completed the questionnaire weekly during treatment and for 6 weeks post treatment. Patients' age ranged from 25 to 65 years with a mean of 47.7 years and median of 50 years. 13 patients were males and 10 were females.

Twenty patients had primary brain tumors and 3 patients had metastatic disease (Figure 2). Two patients had metastases from primaries in the breast while one had from testes. Of the 20 patients with primary brain malignancies, 1 patient had primary central nervous system melanoma, one had papillary tumor of pineal region and 18 had gliomas, of which one was low grade and 17 were high grade gliomas.

The Karnofsky Performance Scale (KPS) ranged from 70 to 90. Twelve patients had a KPS of 90 while 5 had a KPS of 80 and 6 had a KPS of 70 .

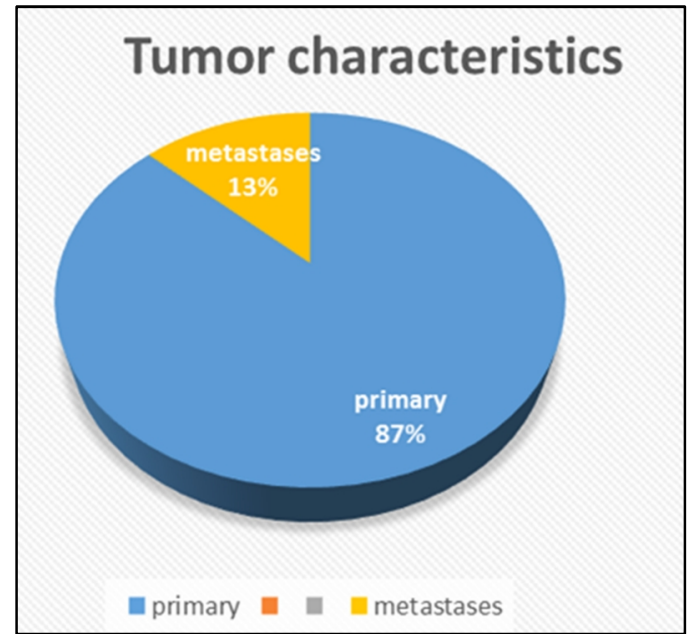

FIG. 2: Pie chart showing tumor characteristics of the patients.

\section{Assessment of somnolence}

Of the 23 patients (Figure 3), 14 patients $(60.86 \%)$ had increased daytime sleepiness. Three out of these 14 (21.4\%) patients were severely sleepy (ESS score: 16-24). 


\section{Excessive sleepiness as per ESS scores}

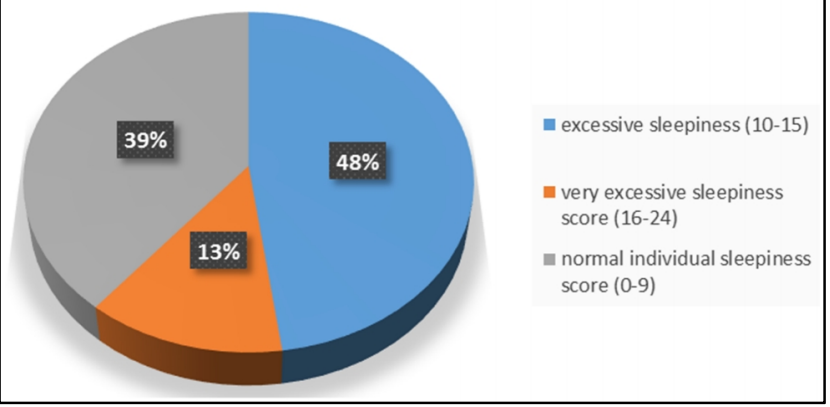

FIG. 3: Pie chart showing patients sleepiness ESS scores.

One out of the 3 patients (33\%) with brain metastases developed somnolence. In the remaining 13 sleepy patients who had primary brain tumors, increase in ESS scores occurred at 5- 6 weeks post radiotherapy in 9 patients and earlier in the remaining 4 patients ( 3 patients in $2^{\text {nd }}$ week and 1 patient in $4^{\text {th }}$ week).

\section{Discussion}

Somnolence syndrome is an early-delayed side effect of radiation treatment to the brain. The condition was first documented by Druckmann in 1929, when treating children for ringworm of the scalp with $150 \mathrm{kV} \mathrm{X}$-ray. ${ }^{5}$ It has generally become recognised as a condition affecting children with acute lymphocytic leukemia (ALL) after receiving prophylactic cranial irradiation. 6,7 However, studies have begun to report that somnolence also appears in adults after irradiation for primary brain tumours. ${ }^{2,3}$

In the present study, we have found an incidence of excessive sleepiness (one of the symptoms of somnolence syndrome) in $60.86 \%$ of the individuals, which is in concordance the $62.9 \%$ incidence reported by Parker et al. ${ }^{8}$ in children receiving cranial irradiation. In the latter study, EEG changes like pathological symmetric slowing of the dominant rhythm and excessive diffuse theta components were used as a method to detect presence of somnolence.

Faithfull S and Brada $\mathrm{M}$ reported incidence of somnolence syndrome in 16 of 19 patients (84.2\%). ${ }^{2}$ This is considerably higher than our reported findings. They have used a prospective diary using visual analog scales of common somnolence symptoms and fatigue. They found that although patients experienced increased drowsiness, lethargy, clumsiness and slow mental processes, they did not require more sleep. Patients commented on the symptoms being 'a sensation that was mentally disabling and a disruption to physical activity by an overwhelming feeling of exhaustion'. Assessment of other symptoms along with propensity to sleep could have resulted in the higher somnolence rates in their study.
The timing of somnolence correlates well with other reported literature; i.e., the symptoms most commonly begin at 5-6 weeks post radiation therapy. ${ }^{9}$ However Faithfull and Brada reported a distinct pattern. ${ }^{2}$ They found that all patients in their study developed symptoms two weeks post-treatment completion. Symptoms then resolved only to resurface in the fifth week, lasting two to five days. Like all other studies, such a bimodal presence of somnolence was not found in our study.

Epworth Sleepiness Scale is a validated eight-item questionnaire wherein the subject is asked to rate on a four point scale (0-3) his/her likelihood of falling asleep in 8 different situations. Scores range from 0 (the least sleepy) to 24 (the most sleepy). The higher the score, the higher the level of persons' daytime sleepiness. Scores equal to or above 10 are interpreted as increased daytime sleepiness.

The Epworth Sleepiness Scale has been validated in sleep disorders using Multiple Sleep Latency Test. ${ }^{3}$ Multiple sleep latency tests measure the propensity to fall asleep when the patient is given $4-5$ programmed 20 to 40 minute nap opportunities 2 hours apart during daytime with EEG, EOG and EMG monitoring. In our study, sleep latency monitoring has not been done.

The use of Epworth Sleepiness Scale in assessing somnolence syndrome in patients receiving radiation therapy to brain is a novel approach and has been used in in only one previously reported study in the surveyed literature till date. Azita Gilardi from University of California- Los Angeles has used Epworth Sleepiness Scale along with other scales and an actigraphic watch to measure somnolence. ${ }^{10}$ It is an easily available and a simple questionnaire. Besides, it can effectively grade the severity of somnolence based on the scores being 11-15 and 16-24.

The major limitation of using the ESS alone in assessing radiation induced somnolence, is that it can detect only increased sleepiness from the spectrum of symptoms encompassing this entity, which can range from mild drowsiness to extreme exhaustion, low-grade fever, nausea, anorexia and headache. Besides, the self-scoring and gradation of scoring in the ESS introduce an element of subjectivity. Hence, using the Epworth Sleepiness Scale along with assessment of common symptoms of somnolence syndrome and addition of an objective tool like Littman scale ${ }^{6}$ will be more accurate in making a definitive diagnosis of somnolence syndrome.

\section{Merits of the study}

It is a prospective study. To the best of our knowledge, it is the first study using Epworth Sleepiness Scale as the sole tool to assess post radiation somnolence syndrome 


\section{Limitations}

The sample size is small and only 3 patients had metastatic brain cancer. Hence, a meaningful comparison of somnolence at various doses could not be undertaken.

\section{Future prospective}

Epworth Sleepiness Scale can be used along with visual analog scale for assessment. Together, the symptomatology will be better understood along with quantification of somnolence.

\section{Conclusion}

Somnolence occurred in $60.86 \%$ of the patients who underwent radiation treatment for brain tumors, which is in accordance with existing literature. Epworth Sleepiness Scale is an effective tool to assess somnolence. However, it assesses for only one of the components of somnolence syndrome. It will be ideal to combine it with scales for assessment of other relevant symptoms.

\section{Conflict of interest}

The authors declare that they have no conflicts of interest. The authors alone are responsible for the content and writing of the paper.

\section{References}

1. Halperin EC, Perez CA, Brady LW. Principles and Practice of Radiation Oncology $5^{\text {th }}$ edition. Philadelphia, Pa: Lippincott Williams \& Wilkins, 2008.

2. Faithfull S, Brada M. Somnolence syndrome in adults following cranial irradiation for primary brain tumors. Clin Oncol (R Coll Radiol) 1998; 10:250-4.

3. Powell C, Guerrero D, Sardell S, et al. Somnolence syndrome in patients receiving radical radiotherapy for primary brain tumours: a prospective study. Radiother Oncol 2011; 100: 131-6.

4. Johns MW. A new method for measuring daytime sleepiness: the Epworth sleepiness scale. Sleep1991;14: 540-5.

5. Druckman, A. Schlafsucht als Folge der Rontgenbestrahlung. Beitrag zur strahlenempfindlichkeit des Gehims. Strahlen-therapie 1929; 33: 382-84.

6. Freeman JE, Johnston PG, Voke JM. Somnolence after prophylactic cranial irradiation in children with acute lymphoblastic leukaemia. Br Med J1973; 4:523-5.

7. Littman P, Rosenstock J, Gale G, et al. The somnolence syndrome in leukemic children following reduced daily dose fractions of cranial radiation. Int J Radiat Oncol Biol Phys 1984; 10:1851-3.

8. Parker D, Malpas JS, Sandland R, et al. Outlook following "somnolence syndrome" after prophylactic cranial irradiation. $\mathrm{Br} \mathrm{Med} J$ 1978; 1:554.

9. Woodford K. Somnolence syndrome after cranial irradiation: a literature review. The Radiographer 2007: 54; 30-33.

10. Gilardi AE, Withers HR. Somnolence induced by radiation therapy of brain tumor. Proceedings of the AACR, Volume 44, $1^{\text {st }}$ ed., 2003. 